\title{
Heurísticos y racionalidad: ¿La ignorancia beneficia a los estudiantes en algún sentido?
}

\section{Heuristic and rationality: does ignorance benefit students in any way?}

\section{Heurísticos e racionalidade: a ignorância beneficia aos estudantes em algum sentido?}

\author{
Miguel López Astorga ${ }^{1}$
}

\begin{abstract}
RESUMEN
Gigerenzer (2007) piensa que la mente humana no es racional, es decir, que no opera siguiendo las prescripciones de la lógica formal. Para demostrarlo, presenta diversos ejemplos del razonamiento de las personas en situaciones cotidianas. En este trabajo, revisamos uno de los ejemplos presentados por Gigerenzer (2007) en este sentido, lo analizamos críticamente y llegamos a dos conclusiones: i) no demuestra que la mente humana es irracional; y ii) nos muestra que la selección de contenidos es un aspecto importante en la práctica pedagógica, pues puede provocar, si no se hace adecuadamente, concepciones excluyentes de determinadas realidades en los estudiantes.

Palabras-clave: contenidos; exclusión; lógica; racionalidad.
\end{abstract}

\footnotetext{
ABSTRACT

Gigerenzer (2007) thinks human mind is not rational, that is to say, it does not work following prescriptions of formal logic. To demonstrate it, Gigerenzer (2007) presents everyday reasoning examples in common

${ }^{1}$ Doctor en Lógica y Filosofía de la Ciencia, Académico del Departamento de Educación, Universidad de Los Lagos, Osorno, Chile.
} 
people. In this paper, we checked an example presented by Gigerenzer (2007) in this way, we analyzed it and we found two conclusions: i) It does not prove that human mind is irrational and ii) it shows that contents selection is an important aspect in pedagogical practice, and that this selection can generate, if it is not done correctly, excludable conceptions about some realities in students.

Keywords: contents; exclusion; logic; rationality.

\section{RESUMO}

Gigerenzer (2007) pensa que a mente humana não é racional, isto é, que não opera seguindo as prescrições da lógica formal. Para demonstrá-lo, apresenta diversos exemplos de raciocínios de pessoas em situações cotidianas. Neste trabalho, revisamos um dos exemplos apresentados por Gigerenzer (2007), o analisamos criticamente e chegamos a duas conclusões: i) não demonstra que a mente humana é irracional; e ii) nos amostra que a seleção de conteúdos é um aspecto importante na prática pedagógica, pois pode produzir, se não se faz adequadamente, concepções excludentes de determinadas realidades nos estudantes.

Palavras-chave: conteúdos; exclusão; lógica; racionalidade.

\section{Introducción}

En el momento presente, asistimos a un debate en el área de la ciencia cognitiva de trascendencia y con importantes repercusiones para el ámbito de la educación. Nos referimos a la discusión planteada en la actualidad acerca de si los seres humanos verdaderamente actúan de manera racional y lógica cuando necesitan extraer conclusiones, pues se puede pensar, por el contrario, que utilizan heurísticos más o menos irracionales para tal fin.

En realidad, parece que este debate comenzó ya a intensificarse hace más de cuatro décadas, cuando el psicólogo Peter Wason $(1966,1968)$ propuso su famosa tarea de selección de las cuatro tarjetas. Ésta es una tarea lógica bastante simple y de, aparentemente, fácil resolución, pero, sorprendentemente, la mayor parte de los participantes que se enfrentan a ella es incapaz de ofrecer la respuesta lógicamente correcta. Desde los primeros momentos de la aparición de esta tarea, ya empezaron a plantearse argumentos a favor de la idea de que este ejercicio de las cuatro tarjetas demostraba de manera absolutamente concluyente que la mente humana ni es racional ni se basa en las reglas o leyes de 
la lógica clásica para ofrecer soluciones a un problema intelectual determinado, sino que se apoya en heurísticos.

La tarea, como decimos, es bastante sencilla. Siguiendo el ejemplo expuesto en López Astorga (2008), podemos decir que el participante ve cuatro tarjetas, una con una ' $A$ ', otra con una ' $C$ ', otra con un ' 2 ' y otra con un ' 3 '. Él sabe que cada una de estas tarjetas tiene un número por un lado y una letra en su otra cara. Lo que tiene que hacer es indicar la tarjeta o las tarjetas que hay que levantar para comprobar si, siempre que aparece una vocal, ésta va acompañada por un número par. Desde el punto de vista lógico, la respuesta correcta coincide con la selección de las tarjetas ' $\mathrm{A}$ ' (para verificar que tiene un número par en su reverso) y ' 3 ' (para asegurarse de que en su otra cara no figura una vocal). No es, por tanto, adecuado elegir la tarjeta ' $C$ ' (tenga un número par o impar en su lado oculto, puede seguir siendo verdad que las vocales van acompañadas por números pares) o la tarjeta '2' (no hay que comprobar si los números pares van acompañados de vocales, sino si las vocales van acompañadas de números pares. Aunque esta tarjeta tuviera ' $C$ ' en su otro lado, la afirmación continuaría siendo verdadera).

El problema de la tarea de selección de las cuatro tarjetas es que el porcentaje más elevado de participantes suele preferir la combinación de las tarjetas ' $A$ ' (correcta) y '2' (incorrecta) y, ante esto, algunos han pensado que el ser humano no es, como hemos indicado, racional. Argumentos en este sentido pueden ser, por ejemplo, el que propuso el propio Wason (1966), quien hablaba de un 'sesgo de verificación' en el pensamiento humano, pues, según él, en vez de elegir las tarjetas adecuadas, los participantes seleccionaban únicamente las que podían representar un caso que confirmaba la afirmación a comprobar (la tarjeta con la vocal podía tener un número par detrás y la tarjeta con el número par podía tener una vocal en su lado opuesto), o el de Evans y Lynch (1973), autores que coincidían con Wason en que la mente humana no parecía seguir lo dictado por los principios lógicos y que discrepaban de él en el tipo de heurístico que, en su opinión, intervenía en el pensamiento de las personas. Estos últimos preferían hablar de un 'sesgo de emparejamiento', según el que los sujetos se inclinaban, de modo completamente contrario a lo que sugeriría la lógica formal, por las tarjetas que se nombraban en la afirmación que había que revisar, i.e., la tarjeta con la vocal y la tarjeta con el número par.

No obstante, lo relevante de este asunto es, para nosotros, que no se trata de un debate cerrado. En la actualidad se sigue discutiendo y argumentando sobre la tarea de selección de las cuatro tarjetas, siendo a este respecto recomendable la lectura de trabajos más o menos recientes como los de Evans (2008), López Astorga (2008a) o Beller (2010) para comprobarlo. Además, a lo largo de todo este tiempo, han surgido otras tareas y ejercicios de razonamiento controvertidos 
en los que, a pesar de ser también tremendamente sencillos en su estructura, los sujetos que intentan solucionarlos suelen errar igualmente en porcentajes considerablemente elevados. Un ejemplo de este tipo de ejercicio bien puede ser el problema de Linda planteado por Tversky y Kahneman (1983), ejercicio en el que, según estos últimos autores, se revela la existencia de un heurístico que ellos denominan 'heurístico de la representatividad'.

El problema de Linda también es bastante simple. Después de describir a Linda en su época de estudiante universitaria y de comentar que fue muy beligerante con las injusticias sociales y que participó, incluso, en protestas antinucleares, se le pide al participante que ordene, en función de su probabilidad, diferentes opciones de la situación de Linda cuando ella cuenta con treinta y un años. La mayor parte de esas opciones suelen ser distractores y las importantes a menudo son semejantes a éstas: i) Linda trabaja en un banco; y ii) Linda trabaja en un banco y participa en un movimiento feminista. La dificultad aquí es que altos porcentajes de participantes señalan que i) es menos probable que ii), lo cual va en contra de lo establecido como más elemental en la teoría de la probabilidad. Decir que i) es menos probable que ii) es afirmar que un acontecimiento simple es menos probable que un acontecimiento compuesto que incluye, entre sus elementos constitutivos, al acontecimiento simple.

El heurístico de la representatividad defendido por Tversky y Kahneman (1983) supone que la mente humana ignora en sus razonamientos a los principios lógico-matemáticos y que juzga a ii) más probable que i) porque ii) describe una situación más representativa del perfil de Linda. Sin embargo, también es éste un problema sobre el que se sigue reflexionando en el presente y para el que no existe una explicación aceptada por la mayor parte de los autores con respecto a sus dificultades. Son ilustrativas, en este sentido, las lecturas de trabajos como los de Díaz (2005) o López Astorga (2009).

Y es que las polémicas en ejercicios como la tarea de selección de las cuatro tarjetas o el problema de Linda son intensas en la actualidad, como decimos, en el ámbito académico, ya que, a pesar de que algunos se manifiestan, como también acabamos de ver, completamente convencidos de que revelan la existencia de heurísticos en la mente humana, otros creen, como puede comprobarse en algunas de las referencias citadas, que el comportamiento de los participantes en estas pruebas, en realidad, no es tan irracional. En algunos trabajos (LÓPEZ ASTORGA, 2008a, 2009) se plantea incluso que estos ejercicios de razonamiento no demuestran que el ser humano no sea lógico, pues son interpretables en varios sentidos, siendo esta posibilidad de entender en direcciones diversas la labor que hay que realizar la fuente de los errores en las respuestas de los sujetos.

No cabe la menor duda de que esta problemática es interesante en el plano de la educación. Generalmente, el docente pretende desarrollar capacidades, 
destrezas y habilidades en el estudiante y, para hacerlo de manera eficiente, debe conocer cuáles son verdaderamente las capacidades, destrezas y habilidades que realmente se utilizan cuando se desea resolver problemas intelectuales. Puede ocurrir, por ejemplo, que, pretendiendo conseguir mejores resultados en una actividad académica determinada, trabajemos con el alumnado una capacidad que aparentemente se encuentra implicada en dicha actividad, pero que, sin embargo, no lo está. Esto podemos verlo con claridad si pensamos, paralelamente, que algunos de los autores citados sostienen, de modo más o menos explícito, que, aunque reforcemos previamente el ámbito del razonamiento lógico-matemático en sujetos que van a participar en problemas como los mencionados, la tarea de selección de Wason $(1966,1968)$ y el problema de Linda de Tversky y Kahneman (1983), tales sujetos no ejecutarán los mencionados problemas de un modo más óptimo, pues no está claro que dicho ámbito sea el que intervenga con mayor protagonismo en problemas de esta índole.

Pero no son los problemas nombrados, ni algunos otros a los que también podríamos recurrir como ejemplos, los que van a constituir el principal objeto de análisis de estas páginas. Nosotros nos vamos a centrar en otro ejercicio descrito por Gigerenzer (2007) y que, desde su punto de vista, según se puede interpretar, es uno de los que finaliza el debate que acabamos de plantear, puesto que prueba de manera definitiva que la mente humana no opera en función del razonamiento lógico, sino que ella se ve condicionada por reglas adaptativas que se manifiestan en los heurísticos que utilizan las personas cuando piensan.

Nuestro objetivo, empero, es mostrar que este ejercicio al que apela Gigerenzer (2007) realmente no demuestra que la mente del ser humano esté gobernada principalmente por la acción de heurísticos irracionales y que es posible presentar una explicación alternativa de los motivos por los que los participantes en él ofrecen respuestas tan sorprendentes, explicación que, además, se basa en principios fundamentales de la lógica clásica, lo que significa que, en definitiva, se da un razonamiento lógico subyacente en los sujetos cuando tratan de resolverlo.

Con esto no pretendemos decir que el ejercicio al que estamos haciendo referencia sea absurdo o que no proporcione ninguna información relevante. La tarea en la que estamos pensando nos va a evidenciar, por el contrario, hasta qué punto es vital una acción por parte del docente a la que no siempre se le presta la atención adecuada y que no es otra que la selección de contenidos conceptuales. Efectivamente, el ejercicio que vamos a analizar a continuación tiene importantes consecuencias pedagógicas, pues, entre otros aspectos a considerar, nos revela la trascendencia que posee el decantarse por unos contenidos y obviar otros en la planificación de una clase. Vamos, pues, sin más, a exponer cuál es este problema al que estamos aludiendo. 


\section{Detroit y Milwaukee: ¿Cuál de estas dos poblaciones cuenta con un mayor número de habitantes?}

Gigerenzer (2007) presenta como uno de los ejercicios que demuestran claramente que los seres humanos nos guiamos en nuestra actividad intelectual más por heurísticos que por inferencias lógicas rigurosas el siguiente:

Se trata de imaginar que se está participando como concursante en un programa televisivo y que se está a punto de ganar un millón de dólares. Para conseguir tal premio, tan sólo hay que responder una pregunta:

"Which city has the larger population, Detroit or Milwaukee?"” (GIGERENZER, 2007, p. 7).

Según Gigerenzer (2007), no es posible deducir lógicamente la respuesta correcta a esta pregunta. Es necesario recurrir al propio conocimiento general que uno mismo puede poseer para contestarla adecuadamente. No obstante, ello significa que la pregunta es verdaderamente compleja, pues la mayor parte de las personas no conoce con certeza los datos geográficos exactos de estas dos ciudades.

El mismo autor nos cuenta que, junto a Daniel Goldstein, propuso este ejercicio a estudiantes norteamericanos. Un cuarenta por ciento de ellos respondió que la ciudad con mayor población era Milwaukee, mientras que el resto se inclinó por Detroit. El dato sorprendente, empero, fue que replicaron el ejercicio con estudiantes alemanes que cursaban niveles educativos equivalentes y se encontraron con que prácticamente todos ellos votaron por Detroit, es decir, ofrecieron la respuesta correcta.

A partir de estos resultados, se pueden barajar, como nos indica Gigerenzer (2007), diversas hipótesis, como, por ejemplo, que los estudiantes alemanes conocen la geografía de Estados Unidos mucho mejor que los propios estudiantes norteamericanos. Sin embargo, según nos cuenta, ocurría justamente lo contrario, puesto que los estudiantes alemanes sabían muy poco (mucho menos que los estadounidenses) acerca de Detroit y un escaso número de ellos había oído hablar alguna vez de la ciudad de Milwaukee. Parece ser, por consiguiente, que los alemanes acertaron por intuición, siendo la pregunta que se plantea Gigerenzer

\footnotetext{
2 ‘¿Qué ciudad tiene una población mayor, Detroit o Milwaukee?’
} 
(2007) qué es lo que hizo a las intuiciones de los alemanes tan poderosas como para no equivocarse.

Responder a esta pregunta no es, sin embargo, difícil desde su punto de vista. Basta apelar al heurístico de reconocimiento y argumentar que los estudiantes alemanes infirieron que la ciudad que tenía mayor población era la que reconocieron. Los estudiantes norteamericanos no pudieron pensar, nos dice, de este modo, ya que ellos habían oído hablar de las dos ciudades y sabían demasiado. Es en este sentido en el que, en su opinión, puede suponerse que un cierto grado de ignorancia puede llegar a ser beneficioso. Naturalmente, el heurístico de reconocimiento no es infalible y no nos garantiza el éxito siempre. No obstante, en muchos casos se torna bastante útil, pues, a juicio de Gigerenzer (2007), el instinto de optar por lo conocido es muy valioso para la supervivencia en el mundo natural, y ello lo saben bien los publicistas, quienes consideran que los potenciales clientes tienden a inclinarse por aquellos productos conocidos antes que por los desconocidos.

En este sentido, Gigerenzer (2007) nos cuenta que, en su opinión, la intuición, en incontables ocasiones, es la que guía la vida. La inteligencia con frecuencia no se apoya, nos dice, en el pensamiento consciente. El ejemplo que nos proporciona con respecto a estos planteamientos es el relativo a que un hablante nativo de un idioma puede decirnos inmediatamente si una sentencia es correcta desde el punto de vista gramatical o no, pero es mucho más complejo para él expresar verbalmente los principios gramaticales subyacentes en virtud de los cuales una sentencia puede ser correcta o incorrecta en su lengua. Por ello, Gigerenzer (2007) afirma que sabemos mucho más que lo que somos capaces de expresar.

De esta manera, nos comenta que, desde su óptica, una intuición es un juicio que cumple estas tres condiciones:

1.- Aparece rápidamente en la conciencia;

2.- No estamos completamente seguros de sus razones subyacentes;

3.- Es lo suficientemente fuerte como para inducir a la acción.

La intuición es, por tanto, en opinión de Gigerenzer (2007), mucho más que un impulso o un capricho. Está relacionada con heurísticos basados en capacidades evolutivas del cerebro (en el caso del problema descrito, como hemos indicado, en el heurístico de reconocimiento). Sin embargo, creemos importante resaltar que su utilización del término 'evolutivas' no tiene mucha relación con algo producto de la naturaleza ni con algo que se ha consolidado independientemente, sino, según parece, con algo que, a pesar de ser concedido por la naturaleza, ha tenido que ser ejercitado.

En cualquier caso, lo relevante para nosotros es el hecho de que supone que existen mecanismos mentales ajenos a la lógica formal que permiten expli- 
car el comportamiento intelectual humano. Nosotros realmente no sabemos si existen o no tales mecanismos que, según Gigerenzer (2007), se manifiestan en forma de capacidades evolutivas. Lo que sí sabemos, por el contrario, es que los resultados del experimento relativo a las ciudades de Detroit y Milwaukee no demuestran, como pretende Gigerenzer, su existencia. Lo único que nos revela este experimento es lo trascendente que puede llegar a ser seleccionar unos contenidos conceptuales para exponerlos a un grupo de estudiantes y descartar otros, ya que tal acción puede influir en las concepciones globales de la realidad y del mundo que dichos estudiantes se van forjando. Vamos a explicar con detalle y a argumentar sobre estas afirmaciones que acabamos de expresar en lo que sigue. De momento, comenzamos por mostrar que la lógica no es una instancia totalmente ajena al comportamiento de los participantes en el experimento de las dos ciudades descrito.

\section{Revisión crítica del enfoque de Gigerenzer}

Como decimos, los motivos por los que los estudiantes alemanes acertaron en el experimento de Detroit y Milwaukee no son necesariamente irracionales o ilógicos. Es posible, fácilmente, encontrar una cierta lógica subyacente como causa de sus conclusiones, y ello porque no cabe duda, para nosotros, de que los estudiantes alemanes partieron de premisas como éstas:

A.- Si conozco una ciudad extranjera, entonces esa ciudad es importante.

B.- Si una ciudad es importante, entonces tiene una población considerable.

De este modo, para nuestro argumento, basta recordar que una regla lógica básica es la regla del modus ponens, la cual se expresa habitualmente de esta manera en lógica proposicional:

Si $p$, entonces $q$

$p$

$q$

Pensemos, partiendo de las premisas A y B, en la aplicación de esta regla en el problema de Gigerenzer (2007) sobre las dos ciudades. Puesto que Detroit es una ciudad conocida por los estudiantes alemanes, éstos pueden aplicar el modus ponens sin dificultad a la premisa $\mathrm{A}, \mathrm{y}$, así, siendo $\mathrm{p}$ "conocer una ciudad extranjera (en este caso, Detroit)' y correspondiendo q a 'ser una ciudad importante', concluir que Detroit es una ciudad importante. 
Pueden también, claro está, operar de igual forma con respecto a la premisa B. Disponiendo ahora del dato de que Detroit es una ciudad importante, p puede equivaler a 'ser una ciudad importante' y q, por su parte, a 'tener una población considerable'. Es evidente, por tanto, que, mediante este segundo razonamiento, los estudiantes alemanes pueden inferir que Detroit es una ciudad con una población considerable.

Es sencillo notar, no obstante, que dichos estudiantes no pueden realizar las mismas operaciones intelectuales pensando en la ciudad de Milwaukee, ya que Milwaukee, al no ser muy conocida, no puede ser considerada $\mathrm{p}$ en la premisa A, por lo que no es posible operar con razonamientos semejantes.

Como se puede apreciar, cabe interpretar que los estudiantes alemanes razonaron según las prescripciones de la lógica formal, no necesitando procesos inferenciales complejos o artificiosos, sino, simplemente, utilizar una regla bastante básica.

Naturalmente, contra estos argumentos nuestros se puede plantear que, si los admitimos, estamos defendiendo que los estudiantes alemanes no fueron intelectualmente muy rigurosos, pues no siempre las ciudades extranjeras que conocemos son las más importantes, como tampoco las ciudades de importancia son forzosamente las que poseen mayor población (de hecho, una ciudad puede ser importante por su relevancia histórica y no por su número de habitantes). Sin embargo, creemos que no es difícil notar que tales planteamientos no afectarían a nuestra tesis relativa a que es posible interpretar el comportamiento de los estudiantes alemanes como lógico, puesto que la falta de rigor a la que aludirían haría referencia a los procesos de generalización o inducción a partir de los que se obtienen las premisas. Dicho en términos más simples, puede que el participante en este experimento incurra en una generalización al creer, por ejemplo, que sólo son importantes las ciudades que disponen de una determinada población, pero una vez aceptada como premisa esta creencia, su conducta es completamente lógica. El problema no residiría entonces en el razonamiento del sujeto, sino únicamente en los procesos a partir de los que extrae sus conocimientos generales, y ése es otro problema distinto.

Con esto no pretendemos decir que los dictados de la lógica sean las únicas instancias que rigen el pensamiento humano. En este sentido, aceptamos que cabe la posibilidad de que enfoques como los sostenidos, por ejemplo, en López Astorga $(2004,2008$ b) estén en lo cierto y de que sean muy diversos los factores que pueden intervenir en las operaciones mentales humanas, desde aspectos culturales hasta ámbitos emocionales. Entre todos esos factores, como se apunta en trabajos como los citados, la lógica también puede ocupar un lugar, pues el razonamiento lógico bien puede ser uno más de los elementos que caracterizan a la actividad mental humana, aunque, como decimos, no el único. 
En cualquier caso, según entendemos, lo que sí está claro es que existen explicaciones alternativas con respecto a los resultados del experimento de las ciudades de Gigerenzer (2007) y que, si la mente humana opera exclusivamente en base a heurísticos como el de reconocimiento, ello no queda totalmente demostrado por dicho experimento. Quizás una teoría cognitiva más reciente, la teoría dual de razonamiento, pueda ayudarnos a comprender mejor el fenómeno del que nos habla Gigerenzer.

\section{La teoría dual de razonamiento y el problema de las ciudades}

La teoría dual de razonamiento, cuyas bases fundamentales pueden hallarse en trabajos como Evans $(1989,2006,2008)$ o Stanovich $(1999,2004)$, posee, desde nuestro punto de vista, el suficiente alcance explicativo como para proporcionarnos las claves necesarias para entender los resultados obtenidos por Gigerenzer (2007) a los que estamos haciendo referencia en estas páginas.

En líneas generales, la teoría dual de razonamiento distingue dos sistemas cognitivos distintos, denominados, siguiendo la terminología utilizada más frecuentemente y que, según parece, procede de Stanovich (1999), el Sistema 1 y el Sistema 2. El Sistema 1 se encuentra constituido, fundamentalmente, por heurísticos que el sujeto aplica de manera muy rápida y, prácticamente, sin tener conciencia de ellos, mientras que el Sistema 2 se caracteriza, esencialmente, por lentos razonamientos analíticos semejantes a los que tradicionalmente agrupamos como inferencias lógicas.

En un principio, podría suponerse que el debate que nosotros mantenemos aquí con Gigerenzer (2007) se puede expresar, sobre la base del esquema conceptual propuesto por la teoría dual de razonamiento, afirmando que Gigerenzer (2007) defiende que los estudiantes alemanes respondieron a la pregunta sobre cuál de las dos ciudades posee un mayor número de habitantes recurriendo a su Sistema 1 y que nosotros argumentamos que emplearon su Sistema 2. No obstante, la propia teoría dual nos ofrece la posibilidad de acercar las dos posturas y de resolver el aparente misterio. Los partidarios de esta teoría suelen sostener que, si bien algunos subsistemas incluidos en el Sistema 1 poseen un carácter innato, otros se adquieren por el ejercicio, la práctica y la experiencia (resulta muy edificante en este sentido el trabajo de REYNA, 2004). De esta manera, sería consistente con la teoría dual pensar que lo que sucedió con los estudiantes alemanes fue, simplemente, que, por el ejercicio y la práctica, automatizaron actividades lógicas como las descritas en el apartado anterior, las 
cuales corresponden claramente a lo que en la teoría dual se denomina Sistema 2 , hasta transformarlas en un heurístico como el de reconocimiento al que alude Gigerenzer (2007), y que debería incluirse, en el marco de la teoría dual, en el Sistema 1.

Se podría pensar, así, que la teoría dual nos permite compatibilizar la concepción de Gigerenzer (2007) con la explicación lógica ofrecida por nosotros. Sin embargo, tal pensamiento no sería del todo acertado, ya que, si aceptamos las tesis de la teoría dual de razonamiento, no podemos, al mismo tiempo, admitir planteamientos de Gigerenzer (2007) como el relativo a que nunca estamos totalmente seguros de las razones subyacentes de nuestras intuiciones. Y no podemos admitir planteamientos como éste porque dispondríamos de una explicación convincente de por qué utilizamos el heurístico de reconocimiento, la cual no sería otra que la que acabamos de ofrecer en base a la teoría dual de razonamiento y que alude a la automatización de operaciones lógicas inferenciales.

Es, por tanto, obvio, a nuestro juicio, que en la mente humana opera tanto el razonamiento lógico-analítico como los heurísticos y, del mismo modo, que las diferentes facultades o capacidades implicadas en la actividad mental humana no son compartimentos estancos que no mantienen entre sí vínculo alguno. De esta manera, se puede decir, en base a las herramientas conceptuales que nos proporciona la teoría dual, que, incluso cuando recurre a heurísticos, la actividad intelectual del ser humano no se encuentra necesariamente separada de modo tajante de la lógica (véase, sobre este punto, los interesantes argumentos que se presentan, por ejemplo, en STENNING; VAN LAMBALGEN, 2008).

\section{Los contenidos conceptuales y la planificación docente}

Este tema relativo al heurístico de reconocimiento y su origen mental posee, más allá del interesante campo que abre para la metacognición, consecuencias y repercusiones claras para la práctica pedagógica y para la planificación docente. Independientemente de que la explicación precedente basada en la teoría dual de razonamiento sea correcta o no, es evidente que los contenidos conceptuales que se trabajan en el aula con los estudiantes condicionan su comportamiento intelectual.

Esto es algo que hasta Gigerenzer (2007) debe admitir, ya que, mediante el heurístico de reconocimiento, sólo podemos reconocer, valga la redundancia, aquello que ya previamente conocimos. Por tanto, el heurístico de reconocimiento sólo es aplicable a contenidos asimilados, lo que significa que aquellos contenidos conceptuales propuestos por el profesor tendrán muchas más posibi- 
lidades de desencadenar la acción del heurístico de reconocimiento que aquellos de los que el alumno nunca ha tenido noticia.

Una planificación docente se puede realizar en función de diferentes modelos, intentando, por ejemplo, reflejar en ella competencias, objetivos o capacidades a desarrollar. Empero, estas páginas nos han mostrado que un elemento que también debe ser cuidado, y al que no siempre se le presta la debida atención, es el contenido conceptual. Y es que el experimento de las dos ciudades y de su población nos revela que quizás exista una serie de contenidos conceptuales que podemos considerar como básicos y que el estudiante debe conocer para no correr el riesgo de que desconozca determinadas realidades y de que, por consiguiente, les reste importancia.

Con el ejemplo de Detroit y Milwaukee hemos comprobado que las ciudades desconocidas pueden ser consideradas menos relevantes, pero podemos referirnos a muchos más ejemplos tomados de la realidad cotidiana de las aulas. Podemos pensar, por poner el caso, en una clase de filosofía en la que el profesor comenta aspectos fundamentales de las teorías de algunos autores clásicos. El simple hecho de no trabajar otros autores puede provocar que el alumno, si no tiene muchos conocimientos sobre la materia, los considere filósofos menores, con menor producción o con un impacto limitado. Es obvio que un profesor de filosofía en un curso no puede revisar sistemáticamente todos los pensadores de la historia, pero creemos que no debe olvidar nunca, cuando selecciona sus contenidos, que aquellos autores no nombrados pueden ser juzgados como de un estatus intelectual o académico más bajo por los estudiantes. Por ello, debe aclarar continuamente a sus alumnos que su programa se basa en una más de las selecciones posibles y que, perfectamente, se podría haber elegido otros autores.

Lo mismo exactamente puede suceder con los temas trabajados por el profesor de geografía. Las zonas geográficas menos nombradas en los planes de estudio (piénsese, con respecto a esto, en las pocas menciones a los países africanos que aparecen en los programas de algunos sistemas educativos) pueden, fácilmente, por medio de mecanismos cognitivos como los descritos en estas páginas, pasar a un segundo término.

Parece haber, por tanto, exigencias éticas en la selección de contenidos conceptuales, pues, si se elaboran las planificaciones sin el debido rigor y sin una reflexión profunda, se pueden construir esquemas mentales en los alumnos que clasifiquen la realidad de manera injusta o, incluso, discriminatoria. De esta manera, parece cobrar todo su sentido, por ejemplo, la opinión de Mañeru (1999), quien, desde la perspectiva de un enfoque de género, defiende que, cuando únicamente utilizamos en nuestros discursos el género masculino para incluir tanto a hombres como a mujeres, excluimos a estas últimas y las relegamos a un segundo nivel. 
Podemos decir, por tanto, que, en realidad, la ignorancia, lejos de, como se puede concluir de las tesis de Gigerenzer (2007), beneficiar en diversas ocasiones al alumnado, le puede conducir a construcciones mentales de la realidad excluyentes o discriminatorias, y así parecen demostrarlo a nivel cognitivo experimentos como el que hemos analizado en estas páginas.

\section{Conclusiones}

Si la mente humana cuenta con reglas adaptativas que se traducen en la utilización de heurísticos como el de reconocimiento, eso es algo que necesita fundamentarse en mayor medida por medio de más apoyos a nivel teórico y experimental. Creemos que la explicación que hemos ofrecido en este trabajo, que vincula el uso de heurísticos con el ejercicio del razonamiento lógico y que se basa en los planteamientos fundamentales de una teoría tan vigente en la actualidad como la teoría dual de razonamiento, es lo suficientemente concluyente como para, al menos, cuestionar los planteamientos básicos de Gigerenzer (2007), con respecto al heurístico de reconocimiento.

No podemos, según parece, separar tan fácilmente la acción de heurísticos que, aparentemente, parecen irracionales de la inferencia lógica. Ello es algo que no sólo se puede apreciar en estas páginas, sino que, además, se puede observar también en otros trabajos sobre la tarea de selección de Wason o el problema de Linda (por ejemplo, LÓPEZ ASTORGA, 2008a, 2008b, 2009) que apuntan a direcciones compatibles con el enfoque aquí propuesto.

En cualquier caso, lo que parece revelar nuestro estudio sobre el problema de las ciudades y su población de Gigerenzer (2007) sin lugar a dudas es que la elección de contenidos conceptuales en los programas es una actividad que siempre debe ser cuidada en el marco curricular, pues los contenidos que transmitimos a los estudiantes son elementos que inciden decisivamente en la conformación de su conocimiento general.

De este modo, el peligro de crear en la mente del alumnado esquemas de pensamiento poco recomendables nos conduce a vislumbrar cómo el plano cognitivo puede interactuar con componentes éticos y valóricos, ya que, inconscientemente y sin la intención expresa de hacerlo, impartir determinados contenidos puede tener consecuencias no deseadas e incidir en que algunas minorías o ciertos ámbitos culturales, geográficos, sociales, políticos etc. sean marginados, discriminados o ignorados. 
Evidentemente, como apuntamos en líneas precedentes, es imposible plantearle al alumnado que trabaje todos los contenidos posibles de una determinada materia y, por supuesto, no es eso lo que proponemos. Lo que nosotros pensamos, y así también lo hemos reflejado más arriba, es que el profesorado, al trabajar un contenido determinado, debe, si está obviando otros contenidos alternativos que también serían perfectamente válidos, intentar conseguir que el estudiante comprenda que, efectivamente, se trata de una selección basada en criterios que no necesariamente tienen que ver con la importancia, la vigencia o la relevancia de dicho contenido. Esto es particularmente necesario en algunas materias relacionadas con las ciencias sociales y las humanidades en las que el abanico de contenidos posibles para un mismo nivel puede ser bastante amplio.

Así, consideramos que hay que recordar que los valores suponen una instancia transversal en el curriculum y que, como se muestra en este trabajo, la ciencia cognitiva tampoco puede ser ajena a ellos. A veces, se piensa que el razonamiento analítico o lógico presenta pocas relaciones con la reflexión ética. En estas páginas, hemos intentado demostrar que no es así y que, aunque la relación no sea directa, indirectamente es posible observar cómo ciertos mecanismos cognitivos del ser humano pueden conducir a visiones concretas de la realidad que pueden no hacer justicia a la pluralidad y a la diversidad.

\section{REFERENCIAS}

BELLER, S. Deontic reasoning reviewed: psychological questions, empirical findings, and current theories. Cognitive Processing, n. 11, p. 123-132, 2010.

DÍAZ, C. Evaluación de la falacia de la conjunción en estudiantes universitarios. Suma, n. 48, p. 45-50, 2005.

EVANS, J. St. B. T. Bias in human reasoning: causes and consequences. Brighton: Erlbaum, 1989.

. The heuristic-analytic theory of reasoning: extension and evaluation. Psychonomic Bulletin \& Review, v. 13, n. 3, p. 378-395, 2006.

. Dual-processing accounts of reasoning, judgment, and social cognition. Annual Review of Psychology, n. 59, p. 255-278, 2008.

; LYNCH, J. S. Matching bias in the selection task. British Journal of Psychology, n. 64 , p. 391-397, 1973. 
GIGERENZER, G. Gut fellings: the intelligence of the unconscious. Londres: Penguin, 2007.

LÓPEZ ASTORGA, M. Revisión del razonamiento condicional a partir de la tarea de selección. Revising conditional reasoning by means of selection task (Spanish Text). Ann Arbor (Michigan): Proquest, Information and Learning Company, 2004. 78-80, 2008a.

Las cuatro tarjetas y el razonamiento humano. Ciencia Cognitiva, v. 2, n. 3, p. . Relevancia, cuantificación y procesamiento del lenguaje natural. Konvergencias. Filosofía y Culturas en Diálogo, v. VI, n. 19, p. 61-75, 2008 b.

. El problema de Linda y la falacia de la conjunción. Konvergencias. Filosofía y Culturas en Diálogo, v. VII, n. 21, p. 24-43, 2009.

MAÑERU, A. Nombrar en femenino y en masculino. In: LOMAS, C. ¿Iguales o diferentes? Barcelona: Paidos, 1999.

REYNA, V. F. How people make decisions that involve risk: a dual-processes approach. Current Directions in Psychological Science, n. 13, p. 60-66, 2004.

STANOVICH, K. E. Who is rational? Studies of individual differences in reasoning. Mahwah: Erlbaum, 1999.

. The robot's rebellion: finding meaning in the age of Darwin. Chicago: Chicago University Press, 2004.

STENNING, K.; VAN LAMBALGEN, M. Human reasoning and cognitive science. Cambridge: The Massachusetts Institute of Technology Press, 2008.

TVERSKY, A.; KAHNEMAN, D. Extensional versus intuitive reasoning: the conjunction fallacy in probability judgement. Psychological Review, n. 90, p. 293-315, 1983.

WASON, P. C. Reasoning. In: FOSS, B. New horizons in psychology. Harmondsworth (Middlesex): Penguin, 1966.

. Reasoning about a rule. Quarterly Journal of Experimental Psychology, n. 20, p. 273-281, 1968.

Texto recebido em 03 de janeiro de 2011.

Texto aprovado em 26 de setembro de 2011. 